\title{
An Improved Metallicity Calibration with UBV Photometry
}

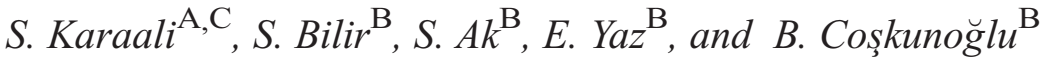 \\ A Beykent University, Faculty of Science and Letters, Department of Mathematics and Computer, \\ Beykent Ayazağa Campus, 34398, Istanbul, Turkey \\ B Istanbul University, Faculty of Sciences, Department of Astronomy and Space Sciences, \\ 34119, Istanbul, Turkey \\ C Corresponding author. Email: karsa@istanbul.edu.tr
}

Received 2010 July 14, accepted 2011 February 9

\begin{abstract}
We used the data of 701 stars covering the colour index interval $0.32<B-V \leq 1.16$, with metallicities $-1.76 \leq[\mathrm{Fe} / \mathrm{H}] \leq+0.40$ dex. The data were taken from the PASTEL catalogue and estimated metallicity-dependent guillotine factors, which provide a more accurate metallicity calibration. We reduced the metallicities of 11 different authors to the metallicities of Valenti \& Fischer (2005), and thus obtained a homogeneous set of data which increased the accuracy of the calibration, i.e. $[\mathrm{Fe} / \mathrm{H}]=-14.316 \delta_{0.6}^{2}-$ $3.557 \delta_{0.6}+0.105$. Comparison of the metallicity residuals for two sets of data based on the metallicitydependent guillotine factors with the ones obtained via metal-free guillotine factors shows that metallicities estimated by means of the new guillotine factors are more accurate than the other ones. This advantage can be used in the metallicity gradient investigation of the Galactic components, i.e. thin disc, thick disc, and halo.
\end{abstract}

Keywords: stars: abundances, stars: metallicity calibration, stars: metal poor

\section{Introduction}

Roman (1955) interpreted the weakness of the metallic lines in the F- and G-type spectra by comparison of the $B-V$ and $U-B$ colours for each star. She stated that an ultraviolet excess ranging from 0.0 to more than $0.2 \mathrm{mag}$, found in most high-velocity stars, is well correlated with the weakness of the lines. Moreover, both anomalies are correlated with velocity, in the sense that the stars with the weakest lines also have the largest ultraviolet excesses and the largest space velocities.

Following Schwarzschild et al. (1955), Sandage \& Eggen (1959) interpreted the observed ultraviolet excess for subdwarfs with the 'blanketing model'. This model predicts that the change in $B-V$ colour index for a given observed ultraviolet excess, $\delta(U-B)$, for $\mathrm{F}$ and $\mathrm{G}$ subdwarfs is sufficient to move most of the subdwarfs with known $M_{V}$ on the Hyades main sequence. The essential point of the theory is that the Fraunhofer lines affect the $U$, $B$, and $V$ regions of the spectrum in different ways so that a weakening of the lines produces changes in the observed colour indices $U-B$ and $B-V$. If the relation between the effect on $U-B$ and $B-V$ is known, then the correction to the observed $B-V$ can be computed from the observed ultraviolet excess. Because the observed $B-V$ for weak-line stars will be bluer than that for strong-line stars of the same temperature, the weak-line stars will fall below the standard main sequence. Therefore, because of the relationship between $\Delta(U-B)$ and $\Delta(B-V)$ we should expect that the displacement of a weak-line star below the standard main sequence will be correlated with the observed ultraviolet excess. Wallerstein \& Carlson (1960) calibrated the ultraviolet excess in terms of [Fe/H] for the first time, and Wallerstein (1962) improved this calibration. The scheme relating the observed ultraviolet excess $\delta(U-B)$ and the blanketing corrections $\Delta(U-B)$ and $\Delta(B-V)$ (for a hypothetical star) are given in Figure 1.

The shapes of the blanketing vectors in the $(U-B)-$ $(B-V)$ diagram are such that stars with different $B-V$ values with the same metal abundances will show different values of ultraviolet excess. For red stars, $\delta(U-B)$ is partially guillotined because the blanketing line is nearly parallel to the intrinsic Hyades line. If the metal abundances are to be compared among stars of different colours, such as in the work carried out for the estimation of the metallicity gradient for the Galactic fields, corrections to the observed $\delta(U-B)$ are needed. Wildey et al. (1962) provided the basis on which normalized ultraviolet excess was computed by Sandage (1969) and Carney (1979). Sandage (1969) gave a procedure to correct guillotine effects on ultraviolet excess values for stars with the same metal abundances but different colours. He plotted 112 stars of large proper motion onto the $(U-B)-(B-V)$ two-colour diagram and compared the $U-B$ colours of maximum abundance with that of Hyades for the same $B-V$ colour. The results are given in Table 1. The columns give: (1) the $B-V$ colour; (2) the Hyades fiducial line; (3) the maximum $U-B$ value for the sample star for the same $B-V$ of the Hyades star; (4) the $\delta(U-B)$ ultraviolet excess of the sample star in 


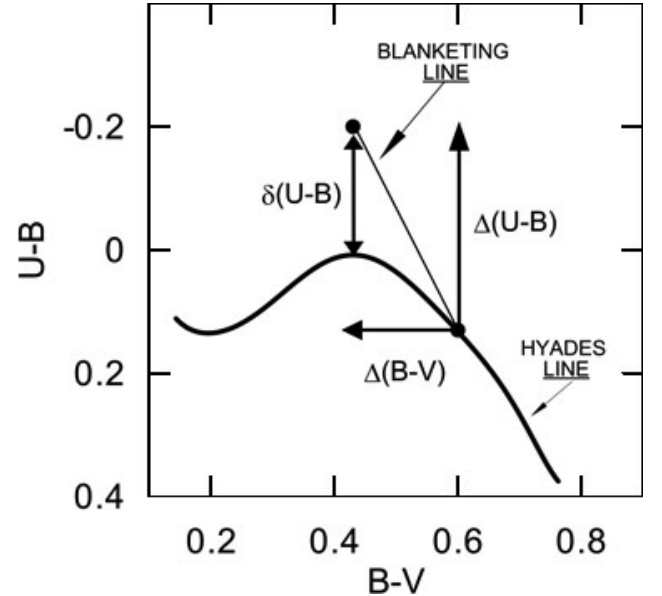

Figure 1 The scheme relating the observed ultraviolet excess, $\delta(U-B)$ and the blanketing corrections $\Delta(U-B)$ and $\Delta(B-V)$ taken from Sandage \& Eggen (1959).

Table 1. The guillotine factors of Sandage (1969). $B-V$ indicates the $B-V$ colour, $(U-B)_{\mathrm{H}}$ the Hyades fiducial line, $(U-B)_{M}$ the maximum $U-B$ value for the sample star for the same $B-V$ of the Hyades star, $\delta(U-B)$ the $\delta(U-B)$ ultraviolet excess of the sample star in question, and $\delta_{0.6} / \delta(U-B)$ the ratio of the excess at $(B-V)=0.60$ (where $\delta(U-B)$ is maximum), $\delta_{0.6}$, to the excess at any other $B-V$

\begin{tabular}{lcccc}
\hline$B-V$ & $(U-B)_{\mathrm{H}}$ & $(U-B)_{\mathrm{M}}$ & $\delta(U-B)$ & $\delta_{0.6} / \delta(U-B)$ \\
\hline 0.35 & 0.03 & -0.22 & 0.25 & 1.24 \\
0.40 & 0.01 & -0.25 & 0.26 & 1.19 \\
0.45 & 0.00 & -0.27 & 0.27 & 1.15 \\
0.50 & 0.03 & -0.25 & 0.28 & 1.11 \\
0.55 & 0.08 & -0.22 & 0.3 & 1.03 \\
0.60 & 0.13 & -0.18 & 0.31 & 1 \\
0.65 & 0.19 & -0.11 & 0.3 & 1.03 \\
0.70 & 0.25 & -0.03 & 0.28 & 1.1 \\
0.75 & 0.34 & 0.08 & 0.26 & 1.19 \\
0.80 & 0.43 & 0.19 & 0.24 & 1.29 \\
0.85 & 0.54 & 0.32 & 0.22 & 1.41 \\
0.90 & 0.64 & 0.44 & 0.2 & 1.55 \\
0.95 & 0.74 & 0.55 & 0.19 & 1.63 \\
1.00 & 0.84 & 0.67 & 0.17 & 1.82 \\
1.05 & 0.94 & 0.79 & 0.15 & 2.06 \\
1.10 & 0.99 & 0.87 & 0.12 & 2.58 \\
\hline
\end{tabular}

question; and (5) the ratio of the excess at $(B-V)=0.60$ (where $\delta(U-B)$ is maximum), $\delta_{0.6}$, to the excess at any other $B-V$. This ratio is defined as the 'guillotine factor' in this paper, i.e. $f_{S}=\delta_{0.6} / \delta(U-B)$, where the subscript $S$ refers to Sandage. Table 1 gives the guillotine factors of Sandage (1969) for a set of 16 colours with $0.35 \leq B-$ $V \leq 1.10$. One can estimate guillotine factors for a larger set of $B-V$ colours by applying an interpolation formula to the data in Table 1. This is the case in some of our works (Karaali et al. 2003; Ak et al. 2007a, 2007b; Yaz \& Karaali 2010).

Carney (1979) normalized the ultraviolet excesses of 101 dwarfs by using the procedure of Sandage (1969) and calibrated them to the metal abundance $[\mathrm{Fe} / \mathrm{H}]$. This calibration could be used to evaluate metal abundances in the $U B V$ photometry. Karaali et al. (2003) improved this calibration by using a different procedure and a different set of $U B V$ data. Other works in different photometries followed the ones carried out in the $U B V$ for metallicity estimation. Buser \& Fenkart (1990) calibrated the $[\mathrm{Fe} / \mathrm{H}]$ metal abundance to the normalized $\delta(U-G)$ excess and $(G-R)$ colour, simultaneously, in the $R G U$ photometry. Strömgren (1966) defined the $m_{1}=$ $(v-b)-(b-y)$ colour difference as a metallicity indicator, where $v, b$, and $y$ are magnitudes for intermediate bands in his $u v b y-\beta$ photometry and the $(B-L)$ colour turned out to be a very sensitive metallicity index for F-G spectral type stars in the VBLUW photometry (Walraven \& Walraven 1960; Trefzger et al. 1995).

There are deviations between the calibrations obtained for the $U B V$ system. Figure 15 of Buser \& Kurucz (1992) compares these calibrations based on empirical data (Carney 1979; Cameron 1985) or theoretical models (Buser \& Kurucz 1978, 1985; Vandenberg \& Bell 1985). The reason for these differences originates from two sources. Firstly, although researchers use the $U B V$ data of the same stars, the references and hence the $U B V$ magnitudes or colours therein may be different. Secondly, different atmospheric parameters may be used by different researchers in estimation of the metallicities used for $[\mathrm{Fe} / \mathrm{H}]-\delta_{0.6}$ calibration (cf. Cayrel de Strobel et al. 2001).

The guillotine factors of Sandage (1969) are colour dependent, but not metallicity dependent. However, the isometallicity lines in the $(U-B)-(B-V)$ two-colour diagram are not parallel to each other for the whole colour range of $B-V$, which indicates the dependence of the guillotine factors on the metallicity. This is the main topic of the paper. The data are presented in Section 2. The guillotine factors and the metallicity calibration are given in Section 3, and finally a short discussion is presented in Section 4.

\section{The Data}

The PASTEL catalogue (Soubiran et al. 2010) is the main source of data for our study. 4259 stars with $4 \leq \log g \leq 5$ and with known metallicity and metallicity errors were selected as main sequence stars from the PASTEL catalogue. 3187 of these stars, that were not displaying variability in their magnitudes and were tagged as 'star', 'star in cluster', and 'high proper motion star' in SIMBAD, were used in the study. To obtain $U B V$ data, we consulted the specialized catalogues which are included in the General Catalogue of Photometric Data (Hauck et al. 1990), which provided the data for 2073 stars.

To calibrate the metallicity more accurately, we selected 11 authors appearing in the PASTEL catalogue (Soubiran et al. 2010), whose databases coincide the most with the 2073 stars in our study. These authors are: Valenti \& Fischer (2005), Sousa et al. (2008), Ramirez \& Melendez (2005), Santos et al. (2004), Fuhrmann (2008), Luck \& Heiter (2006), Mishenina et al. (2004), Nissen 
et al. (2002), Ryan \& Smith (2003), Spite et al. (1996), and Tomkin \& Lambert (1999). From all authors we collected a total of 701 stars, the metal abundances of 472 of which were determined by Valenti \& Fischer (2005). Hence, we reduced all the metallicities to Valenti \& Fischer (2005)'s using the calibrations between the metal abundances of common stars in the work of Valenti \& Fischer (2005) and other researchers. Table 2 gives the star catalogue obtained by this procedure. The errors cited for the metal abundances belong to the original figures. The parallaxes were taken from the newly reduced Hipparcos catalogue (van Leeuwen 2007). The $U B V$ data of stars in Table 2 have been dereddened by the following procedure (Bahcall \& Soneira 1980).

$$
A_{d}(b)=A_{\infty}(b) \times\left(1-e^{\frac{-|d \sin (b)|}{H}}\right)
$$

where $b$ and $d$ are the Galactic latitude and the distance of the star (evaluated by means of its parallax), respectively. $H$ is the scale height for the interstellar dust, which is adopted as $125 \mathrm{pc}$ (Marshall et al. 2006), and $A_{\infty}(b)$ and $A_{d}(b)$ are the total absorptions for the model (Schlegel et al. 1998) and for the distance to the star respectively. $A_{\infty}(b)$ may be evaluated by means of Equation 2 .

$$
A_{\infty}(b)=3.1 \times E_{\infty}(B-V),
$$

where $E_{\infty}(B-V)$ is the colour excess for the model taken from the NASA Extragalactic Database. ${ }^{1}$ Then, $E_{d}(B-V)$, i.e. the colour excess for the corresponding star at the distance $d$, can be evaluated by Equation 2 adapted for distance $d$,

$$
E_{d}(B-V)=A_{d}(b) / 3.1,
$$

and can be used for the colour excess $E_{d}(U-B)$ evaluation:

$$
E_{d}(U-B)=0.72 E_{d}(B-V)+0.05 E_{d}^{2}(B-V) .
$$

Finally, the dereddened colour indices are:

$$
\begin{aligned}
& (B-V)_{0}=(B-V)-E_{d}(B-V) \\
& (U-B)_{0}=(U-B)-E_{d}(U-B) .
\end{aligned}
$$

The reduced ultraviolet excess $\delta_{0.6}$ is evaluated by the following equation which is obtained from the data of 133 stars with $0.575 \leq(B-V) \leq 0.625$ (Table 3$)$ :

$$
\begin{aligned}
\delta_{0.6}= & -0.038(0.005)[\mathrm{Fe} / \mathrm{H}]^{3}-0.163(0.019)[\mathrm{Fe} / \mathrm{H}]^{2} \\
& -0.302(0.017)[\mathrm{Fe} / \mathrm{H}]+0.012(0.004)
\end{aligned}
$$

In this study, Karaali's guillotine factor is denoted by $f_{K}$ and is calculated with $f_{K}=\delta_{0.6} / \delta$.

\footnotetext{
${ }^{1}$ http: //nedwww. ipac.caltech. edu/forms/calculator. html.
}

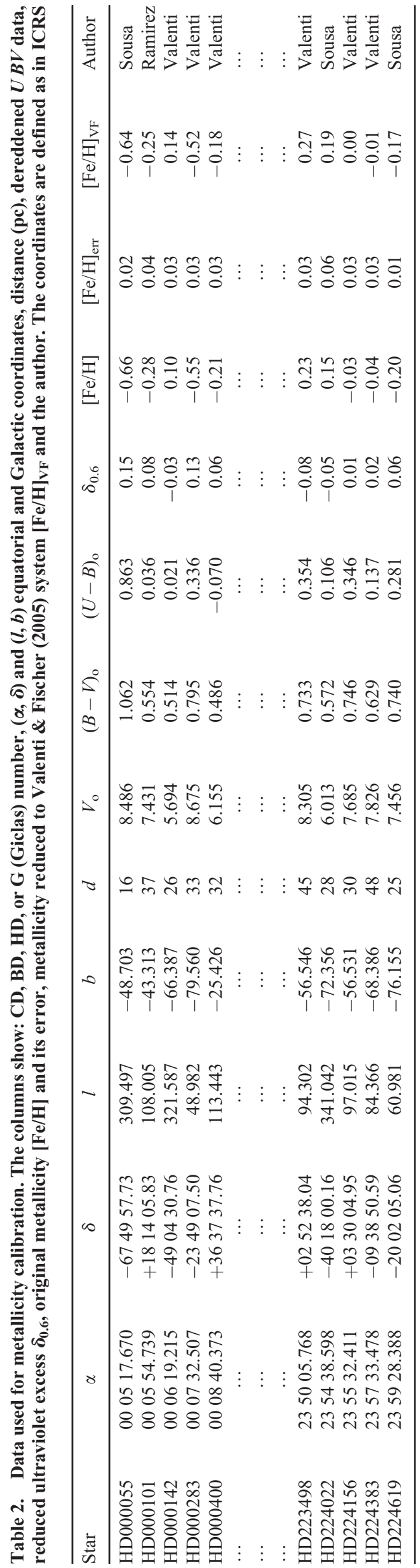




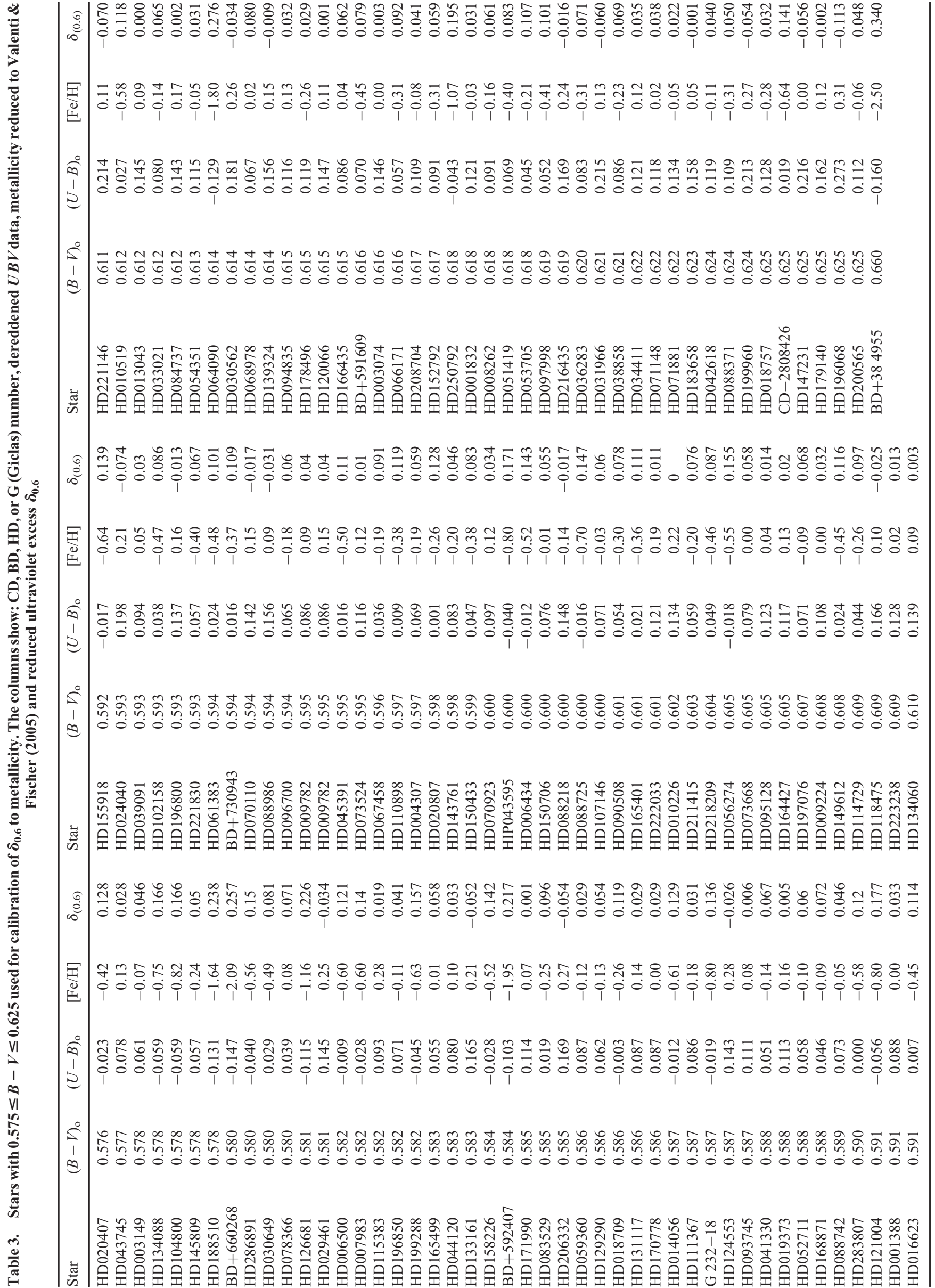




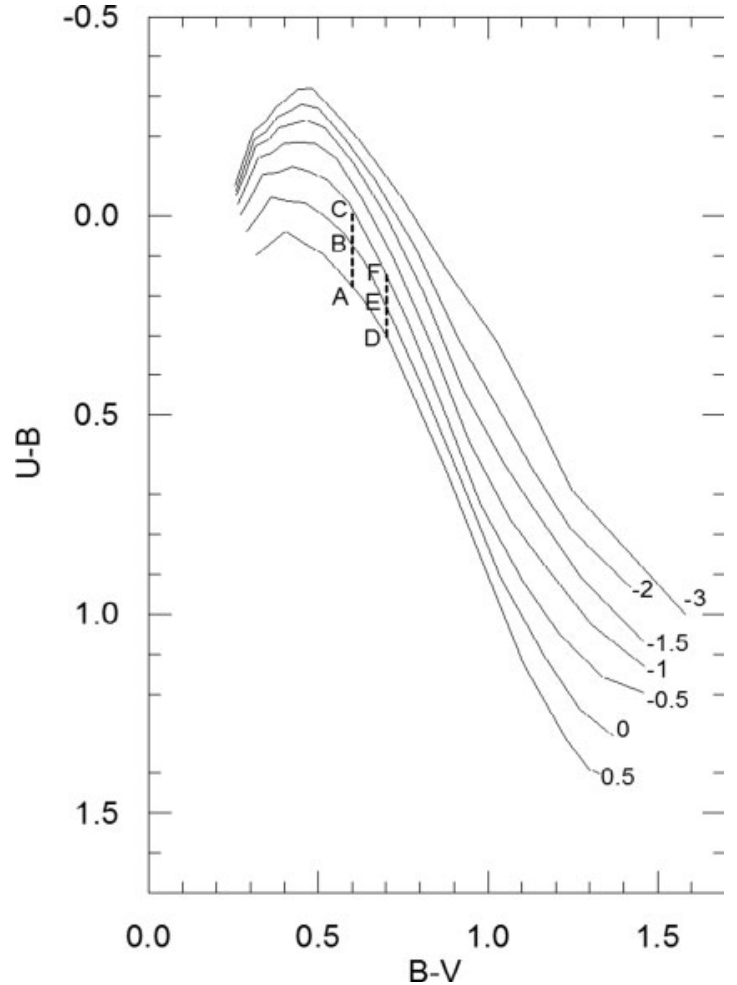

Figure 2 Synthetic isometallicity lines for $U B V$ photometry taken from a stellar model of Lejeune et al. (1997).

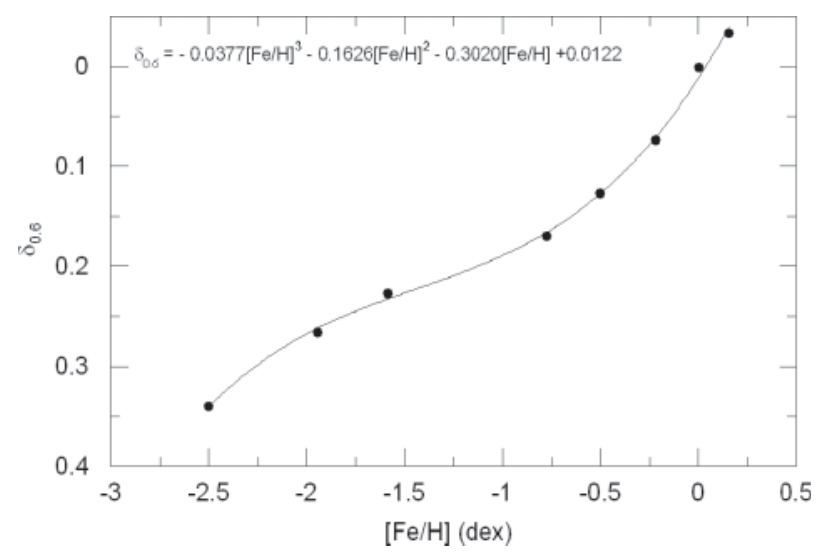

Figure 3 Metallicity versus ultraviolet excess calibration for 133 stars with $0.575 \leq B-V \leq 0.625$.

\section{Methods}

\subsection{New Guillotine Factors}

Sandage (1969) estimated guillotine factors without considering the effect of metallicity. However, Figure 2 shows that the colour gradients for any two different isometallicity lines are not equal to each other, i.e. $\frac{|\mathrm{AB}|}{|\mathrm{DE}|} \neq \frac{|\mathrm{AC}|}{|\mathrm{DF}|}$, which indicates the dependence of guillotine factors on metallicity. Additionally, the $(U-B)_{\mathrm{M}}$ colours in Table 1 correspond to the stars with less metallicity than the Hyades cluster. But the metallicity gradients for the isometallicity line with $[\mathrm{Fe} / \mathrm{H}]=0.5 \mathrm{dex}$ in Figure 2 are rather different from the ones for relatively metal-poor stars, which indicates that guillotine factors for metal-rich stars should be different from those of metal-poor stars of the same $B-V$ colour index.

Then we decided that it should be more appropriate and useful to estimate metallicity-dependent guillotine factors. First, we used 133 stars with colour index $0.575 \leq$ $B-V \leq 0.625$ and calibrated their ultraviolet excess to the metallicity. The calibration (Equation 6) provides ultraviolet excess reduced to $B-V=0.60$ for any star with metal abundance $[\mathrm{Fe} / \mathrm{H}] \geq-2.5 \mathrm{dex}$. Thus, we can use the calibration obtained from Figure 3 to estimate reduced $\delta_{0.6}$ ultraviolet excess for stars with metal abundance $-2.5 \leq[\mathrm{Fe} / \mathrm{H}] \leq 0.15 \mathrm{dex}$.

Next, we separated the stars in Table 2 into eight subsamples with colour indices $0.32<B-V \leq 0.42$, $0.42<B-V \leq 0.49, \quad 0.49<B-V \leq 0.57, \quad 0.57<B-$ $V \leq 0.62, \quad 0.62<B-V \leq 0.69, \quad 0.69<B-V \leq 0.76$, $0.76<B-V \leq 0.85,0.85<B-V \leq 1.16$ and obtained calibrations for the guillotine factors as follows. The number of these colour intervals and their ranges had been decided so as to obtain a constant metallicity gradient for each $B-V$ interval. For example, the ranges for bluer stars, where the metallicity gradient is relatively large, were chosen to be smaller than for the colour interval $0.85<B-V \leq 1.16$, where the metallicity gradient is relatively small. The $(U-B)-(B-V)$ colour diagrams of the whole sample and eight subsamples are shown in Figure 4.

Each subsample was divided into bins and mean $\delta$, $B-V, U-B,[\mathrm{Fe} / \mathrm{H}],(U-B)_{\mathrm{H}}, \delta_{0.6}$, and $f_{K}$ values were evaluated for each subsample (Table 4). A total of 532 stars could be used in the calibration of guillotine factors. The ultraviolet excess $\delta$ of a sample star whose $U-B$ colour index is close to that of a Hyades star of the same $B-V$ colour index is rather small. Hence $f_{K}=\delta_{0.6} / \delta$ becomes rather large for such stars, and they are not reliable. These abnormal $f_{K}$ values may be as large as 20 , for example. Also, we noted that the $f_{K}$ values of some stars were negative. The reason for these unreliable values is errors in the $U-B$ colour index. After rejecting the stars with large and negative $f_{K}$ values, the number of stars was reduced from 701 to 532 . The number of stars used in each bin is given in the last column of Table 4.

The calibration of $\delta$ to $f_{K}$ is given in Figure 5. It is noticeable that the relation of $\delta$ to $f_{K}$ is smooth in all panels, and that the trend of the guillotine factor varies in different panels. In panels (a), (b), (g), and (h), $f_{K}$ assumes its maximum value at intermediate values of $\delta$, whereas in panels (c), (d), (e), and (f) the maximum of $f_{K}$ corresponds to negative values of $\delta$, i.e. metal-rich stars. Figure 6 shows the calibration of $B-V$ to $f_{K}$ for three ultraviolet excesses, $\delta=-0.05,+0.05$, and +0.15 , just to show that one can obtain continuous transitions between colour index and ultraviolet excess. Since Figure 5 is divided according to colours and the fits are in better agreement with data, the obtained equations will be more precise. Therefore, we prefer the equations obtained from Figure 5. 

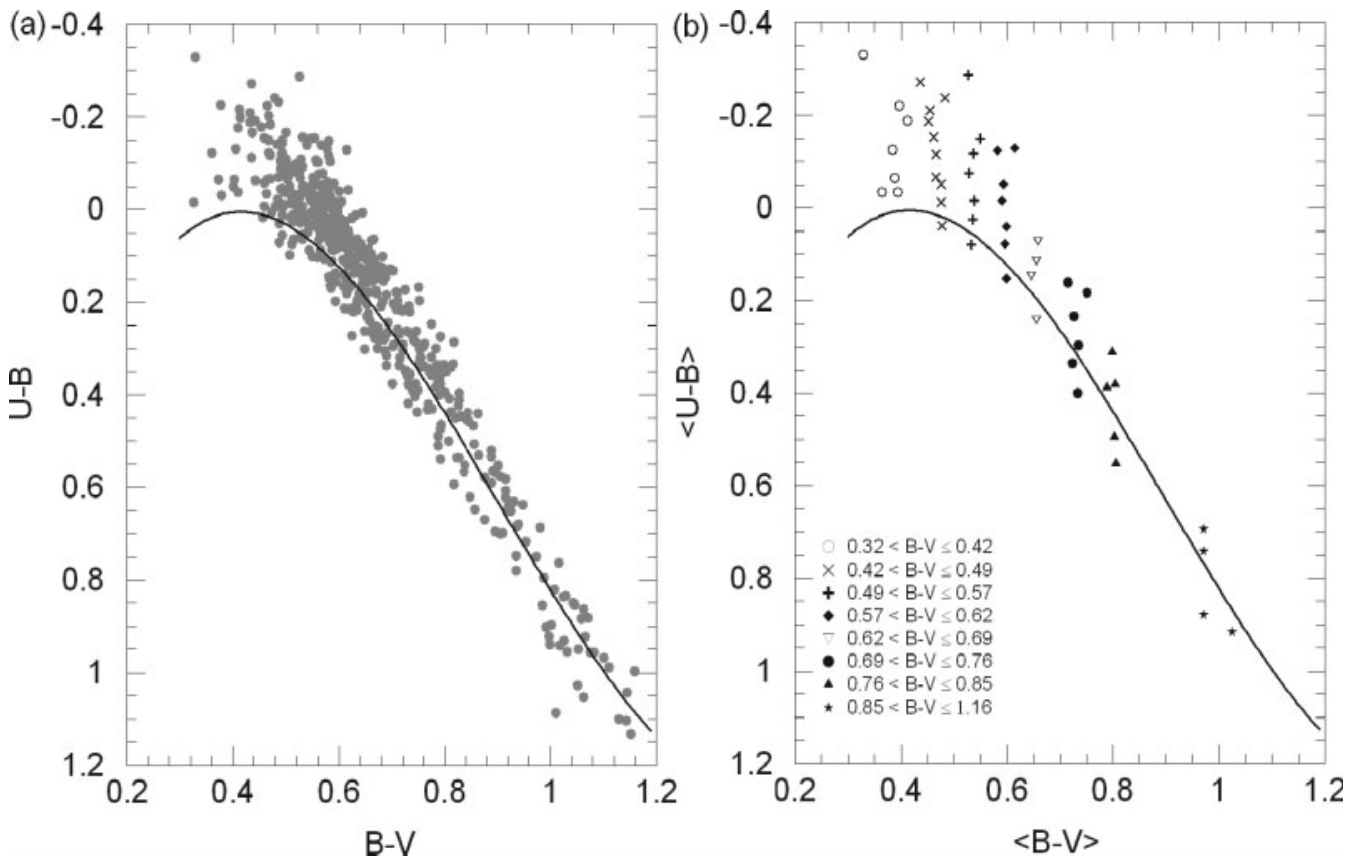

Figure $4(U-B)-(B-V)$ two-colour diagrams for (a) the whole sample, and (b) 50 bins of eight subsamples in Table 4.

Figure 7 shows the distribution of the guillotine factors as a function of metallicity. The lower limit for the guillotine factors of Sandage (1969) is $f_{S}=1$ (Figure 7(b)), corresponding to the colour index $B-V=0.60$, whereas the one estimated in this work may be less than 0.5 , which is not colour dependent, but corresponds to metal abundance $[\mathrm{Fe} / \mathrm{H}] \approx 0$ dex.

\subsection{New Metallicity Calibration}

We used the calibrations in Figure 5 and assigned guillotine factors, $f_{K}$, for 701 stars with metallicity $-1.76 \leq[\mathrm{Fe} / \mathrm{H}] \leq+0.40$ dex. The combination of $f_{K}$ and the ultraviolet excess $\delta$ gives the reduced ultraviolet excess for each star, i.e. $\delta_{0.6}=f_{K} \times \delta$. Then we divided the interval $-0.15 \leq \delta_{0.6} \leq 0.24$ into 11 scans and adopted the centroid of each scan as a locus point to fit a second-order polynomial (Figure 8$)$ to the points $\left(\delta_{0.6}\right.$, $[\mathrm{Fe} / \mathrm{H}])$. The full equation of the polynomial is

$$
\begin{aligned}
{[\mathrm{Fe} / \mathrm{H}]=} & -14.316(1.919) \delta_{0.6}^{2}+3.557(0.285) \delta_{0.6} \\
& +0.105(0.039) .
\end{aligned}
$$

Equation 7 provides metallicities by means of new guillotine factors. We used the guillotine factors of Sandage (1969) and evaluated another set of reduced $\delta_{0.6}$ ultraviolet excesses for the same star sample. Their fit to the corresponding metallicities is given in Figure 8 and in the following formula:

$$
\begin{aligned}
{[\mathrm{Fe} / \mathrm{H}]=} & -11.612(0.496) \delta_{0.6}^{2}+3.419(0.100) \delta_{0.6} \\
& +0.057(0.017)
\end{aligned}
$$

\subsection{Application of the Method}

Now, we have two metallicity calibrations, based on the new guillotine factors estimated in this work and on the guillotine factors adopted from Sandage (1969). We applied these calibrations to two sets of data with $-1.76 \leq[\mathrm{Fe} / \mathrm{H}] \leq 0.4$ dex taken from Karaali et al. (2003) and Karataș \& Schuster (2006) and we compared the evaluated metallicities with the original ones for two calibrations. The metallicities of 75 stars in the first set were estimated spectroscopically, whereas those for 469 stars are based on photometry. There is no overlap between stars in the two sets just mentioned and the sample stars. The results for the data of Karaali et al. (2003) are given in Table 5. The column headings are as follows: Hip No is the Hipparcos number; $\delta$ is the ultraviolet excess; $f_{K}$ and $f_{S}$ are the guillotine factors estimated in this work and adopted from Sandage (1969), respectively; $\delta_{0.6}(K)$ and $\delta_{0.6}(S)$ are ultraviolet excesses reduced by means of $f_{K}$ and $f_{S}$, respectively; $[\mathrm{Fe} / \mathrm{H}]_{o b s},[\mathrm{Fe} / \mathrm{H}]_{K}$, and $[\mathrm{Fe} / \mathrm{H}]_{S}$ are original metallicities taken from the literature and metallicities evaluated via Equations 7 and 8, respectively; $\Delta[\mathrm{Fe} / \mathrm{H}]_{K}$ and $\Delta[\mathrm{Fe} / \mathrm{H}]_{S}$ are residuals for two calibrations, where $K$ and $S$ refer to the data evaluated by means of the guillotine factors estimated in this work and those adopted from Sandage (1969). The results for all 469 stars are not given here in order to save space. However, their statistics are given in Table 6 together with those of 75 stars in the first set.

Comparison of the mean and standard deviations for the residuals of two calibrations for four metallicity intervals, i.e. $-1.76[\mathrm{Fe} / \mathrm{H}] \leq-1,-1<[\mathrm{Fe} / \mathrm{H}] \leq-0.5$, $-0.5<[\mathrm{Fe} / \mathrm{H}] \leq 0$, and $0<[\mathrm{Fe} / \mathrm{H}] \leq+0.4$ dex shows 
Table 4. Ultraviolet excess $\delta$, reduced ultraviolet excess $\delta_{0.6}$ and new guillotine factors $f_{K}$ for each bin of eight sub-samples. $\langle B-V\rangle$ and $\langle U-B\rangle$ are the mean colours, $\langle[\mathrm{Fe} / \mathrm{H}]\rangle$ is the mean metallicity, $\left\langle(U-B)_{\mathrm{H}}\right\rangle$ is the mean Hyades sequence, and $N$ is the number of stars in each bin

\begin{tabular}{|c|c|c|c|c|c|c|c|}
\hline$\langle B-V\rangle$ & $\langle U-B\rangle$ & $\langle[\mathrm{Fe} / \mathrm{H}]\rangle$ & $\left\langle(U-B)_{\mathrm{H}}\right\rangle$ & $\delta$ & $\delta_{0.6}$ & $f_{K}$ & $N$ \\
\hline \multicolumn{8}{|c|}{$0.32<B-V \leq 0.42$} \\
\hline 0.394 & -0.034 & -0.053 & 0.012 & 0.046 & 0.028 & 0.595 & 2 \\
\hline 0.364 & -0.033 & -0.110 & 0.031 & 0.064 & 0.043 & 0.695 & 2 \\
\hline 0.388 & -0.064 & -0.224 & 0.014 & 0.079 & 0.071 & 0.924 & 2 \\
\hline 0.384 & -0.126 & -0.584 & 0.017 & 0.143 & 0.140 & 0.985 & 2 \\
\hline 0.412 & -0.188 & -1.424 & 0.008 & 0.195 & 0.221 & 1.135 & 2 \\
\hline 0.396 & -0.220 & -1.836 & 0.012 & 0.233 & 0.254 & 1.092 & 2 \\
\hline 0.329 & -0.330 & -1.530 & 0.050 & 0.379 & 0.229 & 0.603 & 1 \\
\hline \multicolumn{8}{|c|}{$0.42<B-V \leq 0.49$} \\
\hline 0.477 & 0.039 & 0.082 & 0.018 & -0.021 & -0.014 & 0.819 & 3 \\
\hline 0.475 & -0.012 & -0.054 & 0.017 & 0.029 & 0.027 & 0.964 & 6 \\
\hline 0.475 & -0.051 & -0.228 & 0.017 & 0.068 & 0.073 & 1.082 & 2 \\
\hline 0.466 & -0.066 & -0.307 & 0.014 & 0.08 & 0.090 & 1.152 & 4 \\
\hline 0.466 & -0.115 & -0.633 & 0.014 & 0.129 & 0.145 & 1.119 & 4 \\
\hline 0.462 & -0.153 & -0.981 & 0.013 & 0.166 & 0.187 & 1.128 & 4 \\
\hline 0.452 & -0.186 & -1.268 & 0.01 & 0.196 & 0.210 & 1.072 & 6 \\
\hline 0.455 & -0.211 & -1.443 & 0.011 & 0.222 & 0.223 & 1.003 & 3 \\
\hline 0.482 & -0.237 & -1.763 & 0.019 & 0.256 & 0.249 & 0.973 & 2 \\
\hline 0.436 & -0.271 & -1.745 & 0.007 & 0.278 & 0.244 & 0.879 & 1 \\
\hline \multicolumn{8}{|c|}{$0.49<B-V \leq 0.57$} \\
\hline 0.532 & 0.080 & 0.188 & 0.052 & -0.028 & -0.053 & 1.787 & 12 \\
\hline 0.535 & 0.026 & -0.085 & 0.054 & 0.028 & 0.036 & 1.273 & 24 \\
\hline 0.537 & -0.016 & -0.307 & 0.056 & 0.072 & 0.087 & 1.209 & 38 \\
\hline 0.528 & -0.075 & -0.641 & 0.049 & 0.124 & 0.146 & 1.182 & 27 \\
\hline 0.536 & -0.117 & -1.069 & 0.056 & 0.173 & 0.194 & 1.127 & 10 \\
\hline 0.549 & -0.150 & -1.414 & 0.065 & 0.216 & 0.217 & 1.006 & 4 \\
\hline 0.526 & -0.286 & -1.211 & 0.046 & 0.332 & 0.206 & 0.623 & 1 \\
\hline \multicolumn{8}{|c|}{$0.57<B-V \leq 0.62$} \\
\hline 0.598 & 0.153 & 0.161 & 0.116 & -0.037 & -0.042 & 1.387 & 19 \\
\hline 0.595 & 0.078 & -0.096 & 0.113 & 0.035 & 0.039 & 1.098 & 23 \\
\hline 0.598 & 0.041 & -0.321 & 0.117 & 0.076 & 0.089 & 1.195 & 29 \\
\hline 0.590 & -0.015 & -0.546 & 0.107 & 0.121 & 0.133 & 1.097 & 21 \\
\hline 0.593 & -0.051 & -0.85 & 0.111 & 0.162 & 0.174 & 1.073 & 5 \\
\hline 0.581 & -0.124 & -1.71 & 0.096 & 0.22 & 0.245 & 1.112 & 4 \\
\hline 0.614 & -0.129 & -1.801 & 0.136 & 0.265 & 0.249 & 0.941 & 1 \\
\hline \multicolumn{8}{|c|}{$0.62<B-V \leq 0.69$} \\
\hline 0.655 & 0.243 & 0.201 & 0.192 & -0.05 & -0.057 & 1.274 & 34 \\
\hline 0.645 & 0.147 & -0.111 & 0.179 & 0.032 & 0.042 & 1.288 & 22 \\
\hline 0.655 & 0.115 & -0.276 & 0.193 & 0.078 & 0.081 & 1.038 & 29 \\
\hline 0.658 & 0.073 & -0.611 & 0.196 & 0.123 & 0.143 & 1.177 & 12 \\
\hline \multicolumn{8}{|c|}{$0.69<B-V \leq 0.76$} \\
\hline 0.732 & 0.401 & 0.387 & 0.317 & -0.084 & -0.133 & 1.611 & 6 \\
\hline 0.722 & 0.335 & 0.218 & 0.3 & -0.035 & -0.064 & 1.650 & 13 \\
\hline 0.734 & 0.296 & -0.098 & 0.321 & 0.025 & 0.038 & 1.555 & 11 \\
\hline 0.726 & 0.234 & -0.429 & 0.306 & 0.073 & 0.109 & 1.525 & 17 \\
\hline 0.714 & 0.161 & -0.604 & 0.286 & 0.125 & 0.141 & 1.143 & 4 \\
\hline 0.751 & 0.183 & -0.886 & 0.351 & 0.168 & 0.164 & 0.955 & 2 \\
\hline \multicolumn{8}{|c|}{$0.76<B-V \leq 0.85$} \\
\hline 0.806 & 0.550 & 0.328 & 0.454 & -0.096 & -0.106 & 1.127 & 5 \\
\hline 0.803 & 0.491 & 0.22 & 0.447 & -0.044 & -0.063 & 1.461 & 10 \\
\hline 0.789 & 0.385 & -0.175 & 0.422 & 0.036 & 0.060 & 1.737 & 11 \\
\hline 0.805 & 0.378 & -0.458 & 0.453 & 0.075 & 0.117 & 1.614 & 17 \\
\hline 0.799 & 0.308 & -0.583 & 0.44 & 0.132 & 0.134 & 1.024 & 8 \\
\hline \multicolumn{8}{|c|}{$0.85<B-V \leq 1.16$} \\
\hline 0.972 & 0.878 & 0.36 & 0.782 & -0.097 & -0.121 & 1.332 & 11 \\
\hline 1.025 & 0.915 & 0.215 & 0.877 & -0.038 & -0.062 & 1.627 & 11 \\
\hline 0.971 & 0.741 & -0.165 & 0.776 & 0.035 & 0.055 & 1.539 & 21 \\
\hline 0.971 & 0.693 & -0.373 & 0.777 & 0.083 & 0.100 & 1.214 & 22 \\
\hline
\end{tabular}




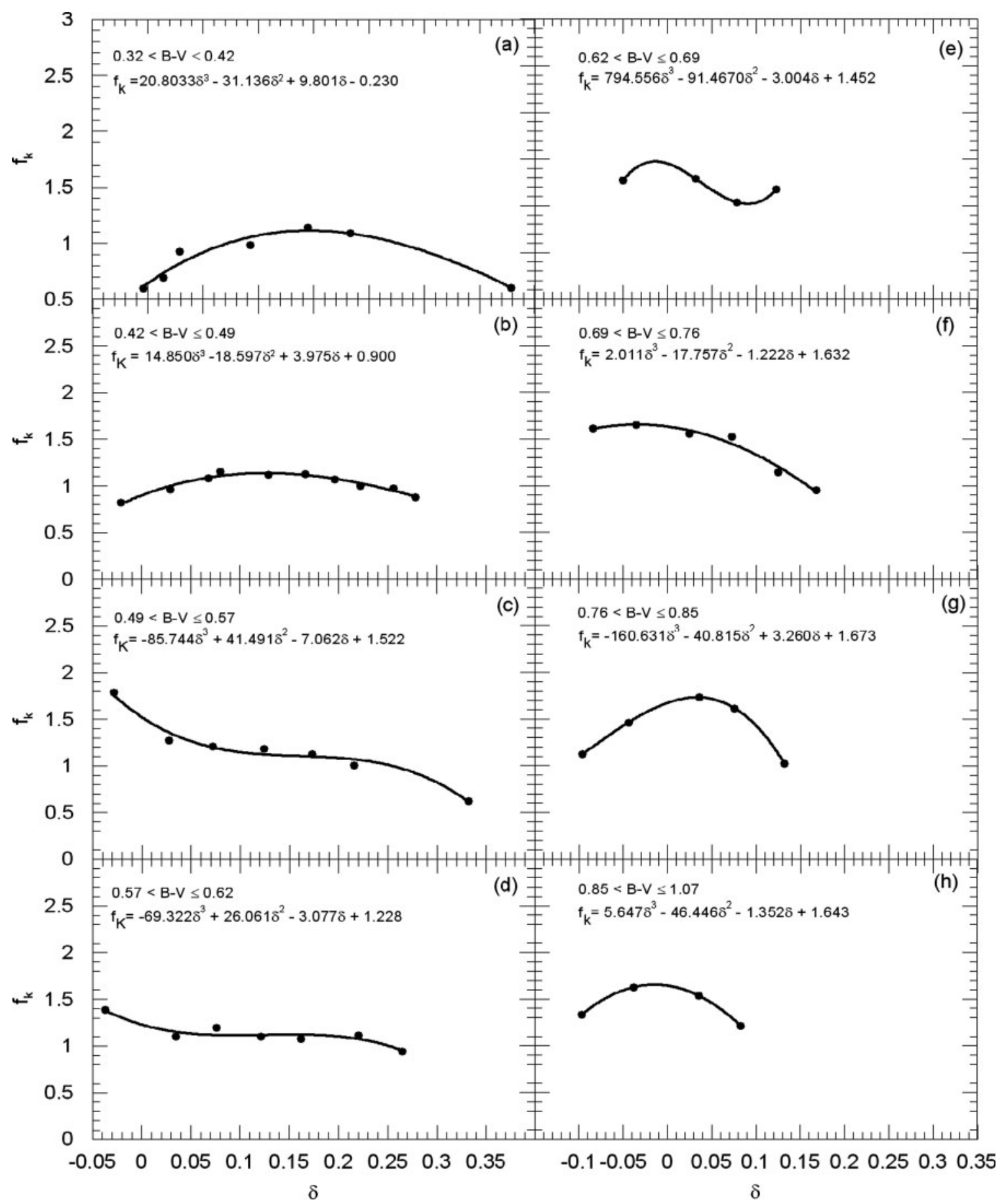

Figure 5 Calibration of ultraviolet excess $(\delta)$ to the guillotine factor $f_{K}$ for eight subsamples.

that there are statistical differences between the two calibrations. In Table 6(a), where the statistics for the first set $(75$ stars) are presented, the agreement is only for the interval $-1<[\mathrm{Fe} / \mathrm{H}] \leq-0.5 \mathrm{dex}$, whereas for other metallicity intervals, the calibration based on metallicitydependent guillotine factors $f_{K}$ is favored. The largest differences between the two sets of statistics occur among the metal-poor stars, i.e. $-1.76<[\mathrm{Fe} / \mathrm{H}] \leq-1 \mathrm{dex}$. In Table 6(b), where the statistics correspond to a larger set of data (469 stars) and where the metallicities were determined photometrically, the agreement between the two calibrations is only for the metallicity interval $-1.76<[\mathrm{Fe} / \mathrm{H}] \leq-1$ dex. The mean deviation of the residuals estimated via guillotine factors $f_{S}$ for the metallicity interval $-0.5<[\mathrm{Fe} / \mathrm{H}] \leq 0$ dex is a bit smaller than the ones estimated via $f_{K}(0.01$ and -0.03 respectively), whereas for the two metallicity intervals $-1<[\mathrm{Fe} / \mathrm{H}] \leq$ -0.5 and $0<[\mathrm{Fe} / \mathrm{H}] \leq+0.4$ dex the mean deviations corresponding to $f_{K}$ are much smaller than those of $f_{S}$.

The comparison of the residuals for all metallicities, i.e. $-1.76<[\mathrm{Fe} / \mathrm{H}] \leq+0.4 \mathrm{dex}$, estimated by means of the two calibrations (Figures 9 and 10) confirms the advantage 


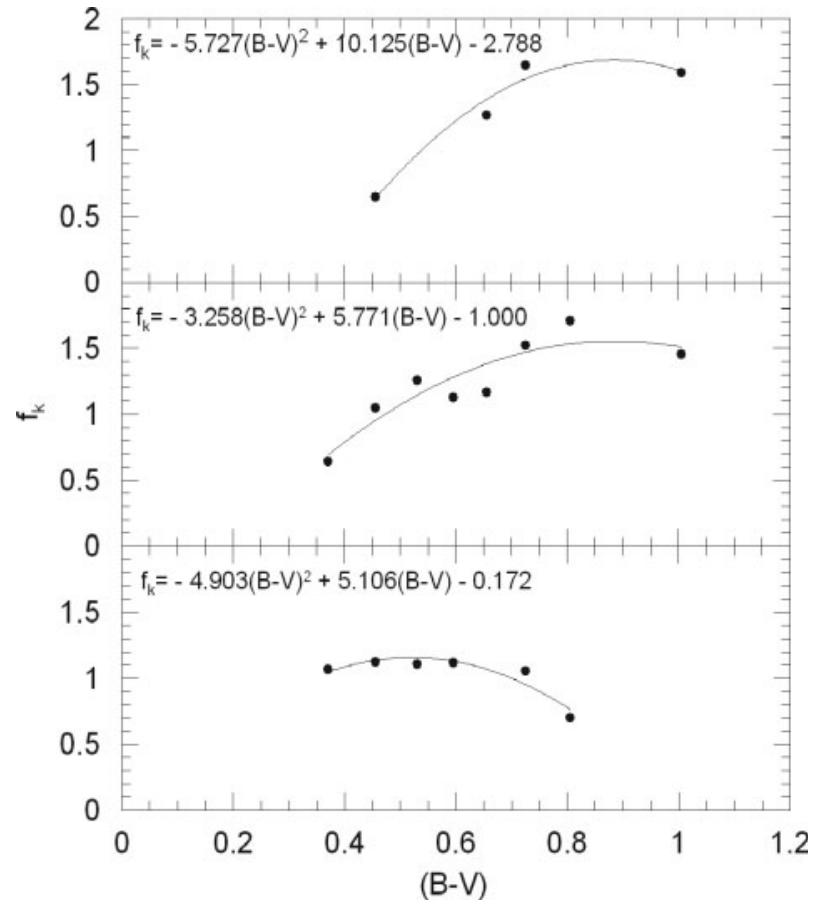

Figure 6 Calibration of $B-V$ colour to the guillotine factor $f_{K}$ for three ultraviolet excesses, $\delta=-0.05,+0.05$, and +0.15 .

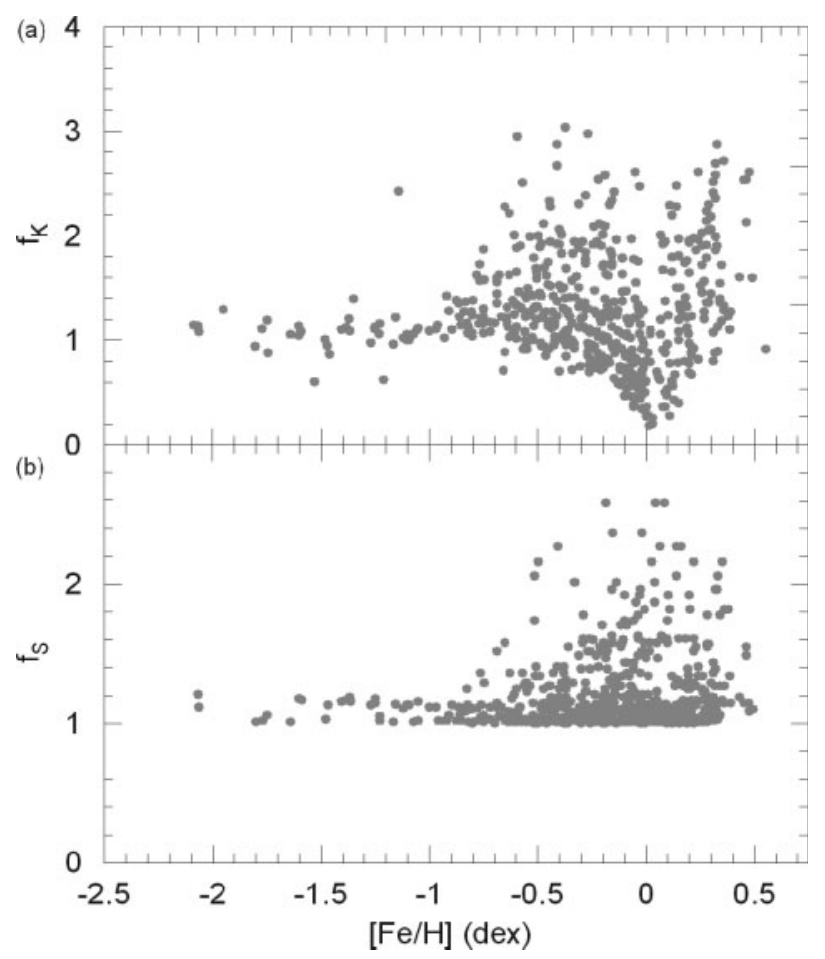

Figure 7 Guillotine factors versus metallicity for (a) metallicitydependent guillotine factors $\left(f_{K}\right)$ and (b) guillotine factors free of metallicity given by Sandage (1969) $\left(f_{S}\right)$.

of the calibration based on metallicity-dependent guillotine factors. There is a small correlation for the residuals in panel (b) in Figure 9, $R^{2}=0.25$, which corresponds to the guillotine factors $f_{S}$, whereas in panel (a), where the residuals were based on the guillotine factors $f_{K}$, the

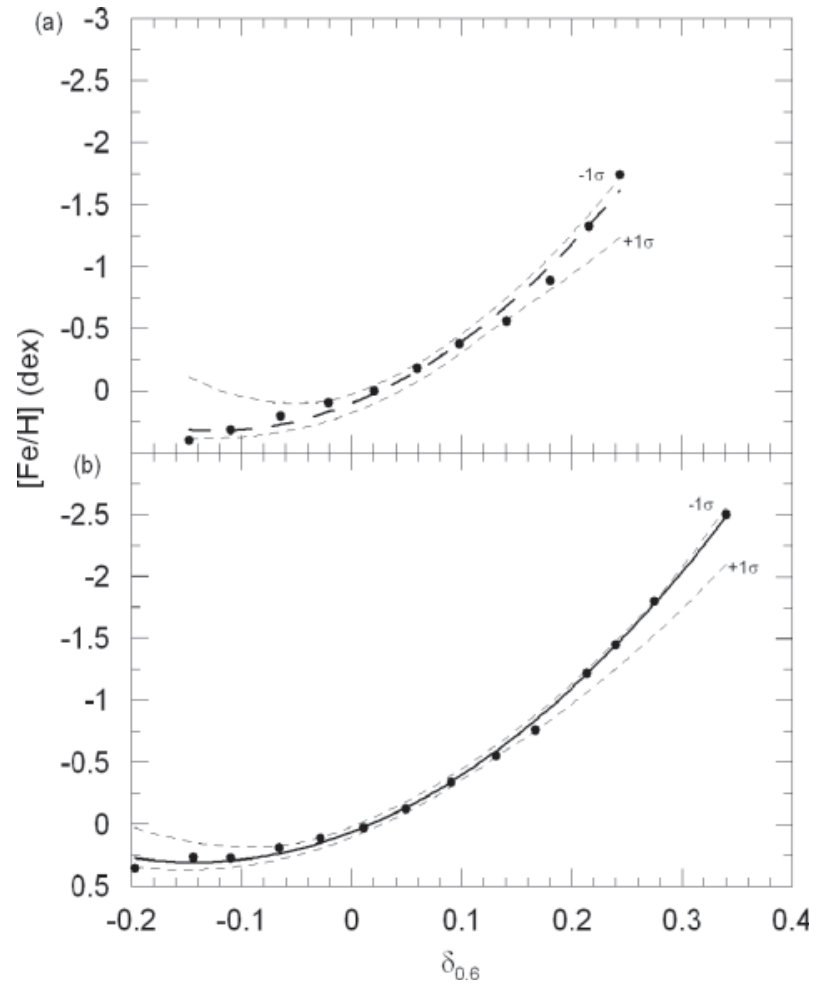

Figure 8 Metallicity calibration based on (a) the metallicitydependent guillotine factors and (b) the guillotine factors adopted from Sandage (1969). The dashed lines denote the $\pm 1 \sigma$ prediction levels.

distribution of the points about the line of zero residual is almost homogeneous, resulting in a zero correlation coefficient, $R^{2}=0.00$.

In Figure 10, the residuals are calibrated to linear equations of the metallicities. The panels (a) and (b) correspond to the residuals estimated via metallicitydependent guillotine factors $f_{K}$ and those of the metal-free ones $f_{S}$. The inclinations of the lines are 0.10 and 0.20 for panels (a) and (b), respectively, favoring the $f_{K}$ factors. Also, the correlation coefficients, $R^{2}=0.03$ and $R^{2}=0.11$, for panels (a) and (b) respectively, confirm our argument. That is, by a smaller correlation coefficient we infer a relatively homogeneous distribution for the residuals in panel (a).

It is interesting that there are small differences in statistics for the two sets of data which can be explained either by the $U B V$ data or metallicities used. We should remember that the metallicities for the first set $(75 \mathrm{stars})$ were estimated spectroscopically, whereas for the second set (469 stars) a photometric procedure was used.

\section{Summary}

We used the data of 11 authors appearing in the PASTEL catalogue (Soubiran et al. 2010) and estimated metallicity-dependent guillotine factors $f_{K}$ which are used in an improved metallicity calibration. The metallicities taken from different authors were reduced to the metallicities of Valenti \& Fischer (2005), so a homogeneous set of metallicities could be obtained. There are differences 
Table 5. Comparison of the original metallicities taken from the literature with those evaluated by using two different calibrations (Equations 7 and 8). Hip No is the Hipparcos number; $\delta$ is the ultraviolet excess; $f_{K}$ and $f_{S}$ are the guillotine factors estimated in this work and adopted from Sandage (1969), respectively; $\delta_{0.6}(K)$ and $\delta_{0.6}(S)$ are ultraviolet excesses reduced by means of $f_{K}$ and $f_{S}$, respectively; $[\mathrm{Fe} / \mathrm{H}]_{o b s},[\mathrm{Fe} / \mathrm{H}]_{K}$, and $[\mathrm{Fe} / \mathrm{H}]_{S}$ are original metallicities taken from the literature and metallicities evaluated via Equations 7 and 8 , respectively; $\Delta[\mathrm{Fe} / \mathrm{H}]_{K}$, and $\Delta[\mathrm{Fe} / \mathrm{H}]_{S}$ are residuals for two calibrations, where $K$ and $S$ refer to the data evaluated by means of the guillotine factors estimated in this work and those adopted from Sandage (1969)

\begin{tabular}{|c|c|c|c|c|c|c|c|c|c|c|}
\hline Hip No & $\delta$ & $f_{K}$ & $f_{S}$ & $\left(\delta_{0.6}\right)_{K}$ & $\left(\delta_{0.6}\right)_{S}$ & {$[\mathrm{Fe} / \mathrm{H}]_{o b s}$} & {$[\mathrm{Fe} / \mathrm{H}]_{K}$} & {$[\mathrm{Fe} / \mathrm{H}]_{S}$} & $\Delta[\mathrm{Fe} / \mathrm{H}]_{K}$ & $\Delta[\mathrm{Fe} / \mathrm{H}]_{S}$ \\
\hline 1599 & 0.085 & 1.112 & 1.01 & 0.095 & 0.086 & -0.26 & -0.362 & -0.325 & 0.102 & 0.065 \\
\hline 3206 & 0.02 & 1.597 & 1.61 & 0.032 & 0.032 & -0.06 & -0.024 & -0.066 & -0.036 & 0.006 \\
\hline 6702 & 0.021 & 0.976 & 1.14 & 0.021 & 0.024 & 0.16 & 0.025 & -0.033 & 0.135 & 0.193 \\
\hline 8102 & 0.086 & 1.397 & 1.14 & 0.120 & 0.098 & -0.38 & -0.528 & -0.389 & 0.148 & 0.009 \\
\hline 10140 & 0.207 & 1.093 & 1.01 & 0.226 & 0.209 & -0.99 & -1.429 & -1.162 & 0.439 & 0.172 \\
\hline 10306 & 0.077 & 0.808 & 1.17 & 0.062 & 0.090 & -0.38 & -0.171 & -0.344 & -0.209 & -0.036 \\
\hline 15330 & 0.077 & 1.042 & 1.02 & 0.080 & 0.078 & -0.20 & -0.271 & -0.282 & 0.071 & 0.082 \\
\hline 17147 & 0.137 & 1.113 & 1.05 & 0.152 & 0.144 & -0.76 & -0.768 & -0.673 & 0.008 & -0.087 \\
\hline 19814 & 0.126 & 1.209 & 1.09 & 0.152 & 0.137 & -0.70 & -0.769 & -0.631 & 0.069 & -0.069 \\
\hline 21272 & 0.025 & 1.727 & 1.23 & 0.044 & 0.031 & -0.03 & -0.078 & -0.061 & 0.048 & 0.031 \\
\hline 22263 & -0.022 & 1.307 & 1 & -0.028 & -0.022 & 0.10 & 0.194 & 0.125 & -0.094 & -0.025 \\
\hline 22596 & 0.087 & 1.112 & 1.01 & 0.096 & 0.087 & -0.32 & -0.370 & -0.331 & 0.050 & 0.011 \\
\hline 27913 & 0.027 & 1.163 & 1.01 & 0.031 & 0.027 & -0.05 & -0.019 & -0.044 & -0.031 & -0.006 \\
\hline 33495 & 0.121 & 1.135 & 1.14 & 0.138 & 0.138 & -0.84 & -0.655 & -0.637 & -0.185 & -0.203 \\
\hline 35377 & 0.107 & 1.055 & 1.02 & 0.113 & 0.109 & -0.38 & -0.477 & -0.453 & 0.097 & 0.073 \\
\hline 36818 & 0.151 & 1.119 & 1.01 & 0.168 & 0.152 & -0.83 & -0.901 & -0.732 & 0.071 & -0.098 \\
\hline 37853 & 0.155 & 1.105 & 1.02 & 0.171 & 0.158 & -0.78 & -0.923 & -0.773 & 0.143 & -0.007 \\
\hline 38541 & 0.251 & 1.002 & 1.01 & 0.251 & 0.253 & -1.76 & -1.692 & -1.553 & -0.068 & -0.207 \\
\hline 38625 & 0.153 & 1.035 & 1.15 & 0.159 & 0.176 & -0.93 & -0.819 & -0.906 & -0.111 & -0.024 \\
\hline 38908 & 0.095 & 1.152 & 1.02 & 0.109 & 0.097 & -0.36 & -0.455 & -0.383 & 0.095 & 0.023 \\
\hline 42438 & 0.073 & 1.115 & 1.01 & 0.082 & 0.074 & -0.27 & -0.282 & -0.260 & 0.012 & -0.010 \\
\hline 43726 & -0.006 & 1.466 & 1.06 & -0.008 & -0.006 & 0.07 & 0.133 & 0.076 & -0.063 & -0.006 \\
\hline 50384 & 0.078 & 1.183 & 1.11 & 0.093 & 0.087 & -0.38 & -0.347 & -0.328 & -0.033 & -0.052 \\
\hline 51248 & 0.068 & 1.117 & 1 & 0.076 & 0.068 & -0.23 & -0.250 & -0.231 & 0.020 & 0.001 \\
\hline 53070 & 0.184 & 1.094 & 1.13 & 0.202 & 0.208 & -1.38 & -1.194 & -1.159 & -0.186 & -0.221 \\
\hline 54772 & 0.197 & 1.075 & 1.17 & 0.211 & 0.230 & -1.15 & -1.287 & -1.344 & 0.137 & 0.194 \\
\hline 56997 & 0.016 & 1.608 & 1.14 & 0.025 & 0.018 & 0.03 & 0.005 & -0.009 & 0.025 & 0.039 \\
\hline 59750 & 0.128 & 1.135 & 1.13 & 0.146 & 0.145 & -0.82 & -0.716 & -0.682 & -0.104 & -0.138 \\
\hline 60632 & 0.227 & 1.018 & 1.17 & 0.231 & 0.265 & -1.68 & -1.478 & -1.666 & -0.202 & -0.014 \\
\hline 62207 & 0.095 & 1.03 & 1.152 & 0.110 & 0.098 & -0.30 & -0.459 & -0.392 & 0.159 & 0.092 \\
\hline 63559 & 0.175 & 1.097 & 1.03 & 0.193 & 0.181 & -0.93 & -1.111 & -0.940 & 0.181 & 0.010 \\
\hline 64394 & 0.015 & 1.186 & 1.01 & 0.018 & 0.016 & 0.06 & 0.035 & 0.001 & 0.025 & 0.059 \\
\hline 64426 & 0.121 & 1.123 & 1.08 & 0.136 & 0.131 & -0.66 & -0.644 & -0.589 & -0.016 & -0.071 \\
\hline 64924 & 0.002 & 1.629 & 1.1 & 0.004 & 0.002 & -0.02 & 0.092 & 0.048 & -0.112 & -0.068 \\
\hline 69972 & -0.036 & 1.631 & 2.01 & -0.059 & -0.073 & 0.26 & 0.266 & 0.245 & -0.006 & 0.015 \\
\hline 70681 & 0.218 & 1.077 & 1 & 0.235 & 0.218 & -1.45 & -1.523 & -1.243 & 0.073 & -0.207 \\
\hline 71681 & -0.01 & 1.652 & 1.47 & -0.017 & -0.015 & 0.14 & 0.162 & 0.106 & -0.022 & 0.034 \\
\hline 71683 & -0.031 & 1.435 & 1.04 & -0.044 & -0.032 & 0.22 & 0.234 & 0.154 & -0.014 & 0.066 \\
\hline 72998 & 0.136 & 1.143 & 1.14 & 0.155 & 0.155 & -0.63 & -0.793 & -0.751 & 0.163 & 0.121 \\
\hline 73005 & 0.093 & 1.495 & 1.27 & 0.139 & 0.118 & -0.55 & -0.665 & -0.508 & 0.115 & -0.042 \\
\hline 75181 & 0.11 & 1.075 & 1.02 & 0.119 & 0.113 & -0.48 & -0.519 & -0.476 & 0.039 & -0.004 \\
\hline 80837 & 0.119 & 1.125 & 1.06 & 0.133 & 0.126 & -0.64 & -0.624 & -0.556 & -0.016 & -0.084 \\
\hline 81800 & 0.037 & 1.315 & 1.05 & 0.048 & 0.039 & -0.01 & -0.100 & -0.092 & 0.090 & 0.082 \\
\hline 82636 & 0.105 & 1.046 & 1.03 & 0.110 & 0.108 & -0.38 & -0.456 & -0.447 & 0.076 & 0.067 \\
\hline 84905 & 0.085 & 1.169 & 1.02 & 0.099 & 0.087 & -0.56 & -0.389 & -0.326 & -0.171 & -0.234 \\
\hline 88745 & 0.121 & 1.123 & 1.08 & 0.136 & 0.131 & -0.42 & -0.644 & -0.589 & 0.224 & 0.169 \\
\hline 89554 & 0.211 & 1.05 & 1.14 & 0.222 & 0.241 & -1.44 & -1.387 & -1.439 & -0.053 & -0.001 \\
\hline 96258 & 0.018 & 0.966 & 1.13 & 0.018 & 0.021 & -0.13 & 0.038 & -0.018 & -0.168 & -0.112 \\
\hline 96901 & -0.001 & 1.454 & 1.04 & -0.001 & -0.001 & 0.08 & 0.108 & 0.059 & -0.028 & 0.021 \\
\hline 97063 & -0.016 & 0.833 & 1.13 & -0.013 & -0.018 & 0.02 & 0.149 & 0.114 & -0.129 & -0.094 \\
\hline 98020 & 0.208 & 1.091 & 1 & 0.227 & 0.208 & -1.37 & -1.443 & -1.160 & 0.073 & -0.210 \\
\hline 99026 & 0.037 & 0.549 & 1.17 & 0.020 & 0.043 & 0.02 & 0.027 & -0.112 & -0.007 & 0.132 \\
\hline 99461 & 0.11 & 1.327 & 1.41 & 0.146 & 0.155 & -0.58 & -0.717 & -0.751 & 0.137 & 0.171 \\
\hline 99889 & 0.05 & 0.642 & 1.19 & 0.032 & 0.059 & -0.05 & -0.023 & -0.185 & -0.027 & 0.135 \\
\hline 100568 & 0.187 & 1.092 & 1.05 & 0.204 & 0.196 & -1.22 & -1.215 & -1.060 & -0.005 & -0.160 \\
\hline 100792 & 0.154 & 1.125 & 1.13 & 0.174 & 0.174 & -0.99 & -0.944 & -0.892 & -0.046 & -0.098 \\
\hline 102011 & 0.047 & 0.622 & 1.17 & 0.029 & 0.055 & -0.03 & -0.010 & -0.165 & -0.020 & 0.135 \\
\hline 102029 & -0.021 & 0.807 & 1.15 & -0.017 & -0.024 & 0.15 & 0.162 & 0.133 & -0.012 & 0.017 \\
\hline 102485 & -0.003 & 0.886 & 1.17 & -0.003 & -0.004 & -0.11 & 0.116 & 0.070 & -0.226 & -0.180 \\
\hline
\end{tabular}


Table 5. (Continued)

\begin{tabular}{lcllrrrrrrr}
\hline Hip No & \multicolumn{1}{c}{$\delta$} & $f_{K}$ & $f_{S}$ & \multicolumn{1}{c}{$\left(\delta_{0.6}\right)_{K}$} & \multicolumn{1}{c}{$\left(\delta_{0.6}\right)_{S}$} & \multicolumn{1}{c}{$[\mathrm{Fe} / \mathrm{H}]_{o b s}$} & {$[\mathrm{Fe} / \mathrm{H}]_{K}$} & {$[\mathrm{Fe} / \mathrm{H}]_{S}$} & $\Delta[\mathrm{Fe} / \mathrm{H}]_{K}$ & $\Delta[\mathrm{Fe} / \mathrm{H}]_{S}$ \\
\hline 102531 & -0.057 & 0.609 & 1.12 & -0.035 & -0.064 & 0.12 & 0.211 & 0.228 & -0.091 & -0.108 \\
103269 & 0.237 & 1.04 & 1.01 & 0.246 & 0.239 & -1.60 & -1.638 & -1.424 & 0.038 & -0.176 \\
103498 & 0.171 & 1.099 & 1.08 & 0.188 & 0.185 & -0.99 & -1.070 & -0.971 & 0.080 & -0.019 \\
104659 & 0.194 & 1.087 & 1.09 & 0.211 & 0.212 & -1.42 & -1.286 & -1.188 & -0.134 & -0.232 \\
105184 & 0.027 & 1.322 & 1.02 & 0.035 & 0.027 & -0.14 & -0.038 & -0.045 & -0.102 & -0.095 \\
105864 & 0.019 & 0.968 & 1.15 & 0.018 & 0.022 & 0.08 & 0.035 & -0.023 & 0.045 & 0.103 \\
109646 & 0.121 & 1.123 & 1.08 & 0.136 & 0.131 & -0.59 & -0.644 & -0.589 & 0.054 & -0.001 \\
110778 & 0.071 & 1.116 & 1.01 & 0.079 & 0.071 & -0.13 & -0.264 & -0.246 & 0.134 & 0.116 \\
110785 & 0.011 & 1.449 & 1.08 & 0.016 & 0.012 & -0.04 & 0.044 & 0.014 & -0.084 & -0.054 \\
110996 & -0.032 & 1.638 & 2.27 & -0.053 & -0.073 & 0.25 & 0.253 & 0.245 & -0.003 & 0.005 \\
113357 & 0.014 & 1.392 & 1.06 & 0.020 & 0.015 & 0.12 & 0.028 & 0.002 & 0.092 & 0.118 \\
113896 & 0.035 & 1.148 & 1.01 & 0.041 & 0.036 & -0.10 & -0.064 & -0.081 & -0.036 & -0.019 \\
114081 & -0.011 & 0.853 & 1.15 & -0.010 & -0.013 & 0.25 & 0.138 & 0.099 & 0.112 & 0.151 \\
114096 & 0.037 & 1.315 & 1.05 & 0.048 & 0.039 & 0.09 & -0.100 & -0.092 & 0.190 & 0.182 \\
114210 & 0.048 & 0.629 & 1.18 & 0.030 & 0.056 & -0.17 & -0.015 & -0.173 & -0.155 & 0.003 \\
116824 & 0.02 & 0.41 & 1.19 & 0.008 & 0.023 & 0.09 & 0.076 & -0.029 & 0.014 & 0.119 \\
\hline
\end{tabular}

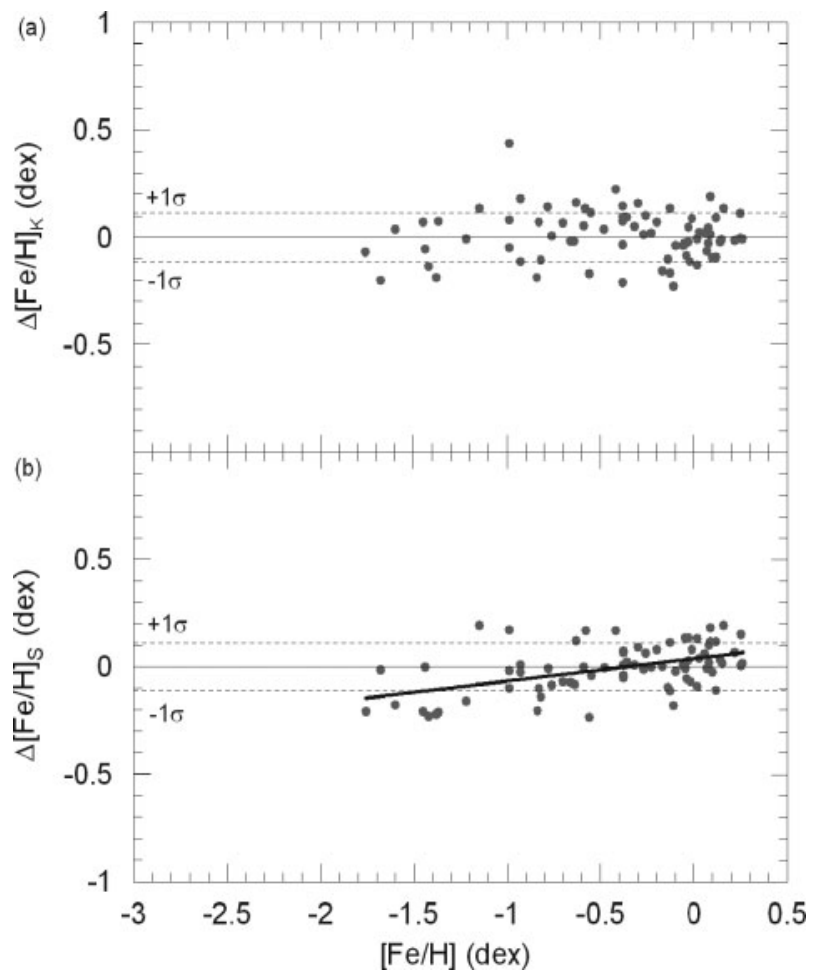

Figure 9 Metallicity residuals versus metallicity for 75 stars taken from Karaali et al. (2003), (a) for the calibration based on metallicitydependent guillotine factors, and (b) for the calibration based on guillotine factors adopted from Sandage (1969). The dashed lines denote one standard deviation.

between the new guillotine factors $f_{K}$ and the factors $f_{S}$ adopted from Sandage (1969).

We derived metallicity calibrations for two sets of guillotine factors using the same procedure and applied them to two different sets of data. The data of the first set were taken from Karaali et al. (2003), whereas those of the second set are from Karataș \& Schuster (2006). For the first set, the mean deviations of the residuals for two calibrations are different. The agreement is only for

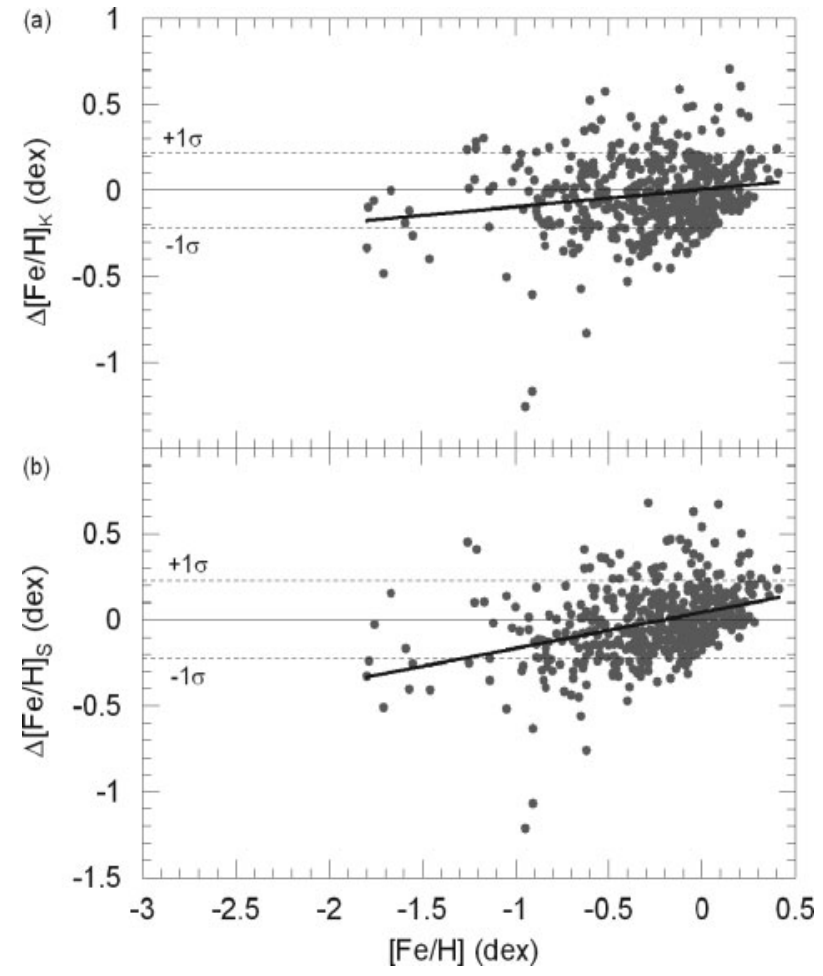

Figure 10 Metallicity residuals versus metallicity for 469 stars taken from Karataş \& Schuster (2006), (a) for the calibration based on metallicity-dependent guillotine factors and (b) for the calibration based on guillotine factors adopted from Sandage (1969). The dashed lines denote one standard deviation. The inclination of the calibration line in the upper panel is less than the one in the lower panel, favoring the metallicity calibration based on metallicity-dependent guillotine factors.

the metallicity interval $-1<[\mathrm{Fe} / \mathrm{H}] \leq-0.5 \mathrm{dex}$, whereas for the metallicity intervals $-1.76<[\mathrm{Fe} / \mathrm{H}] \leq-1,-0.5<$ $[\mathrm{Fe} / \mathrm{H}] \leq 0$, and $0<[\mathrm{Fe} / \mathrm{H}] \leq+0.4$ dex, the mean deviations corresponding to the metallicity dependent guillotine factors $f_{K}$ are much smaller than those estimated via the guillotine factors adopted from Sandage (1969), $f_{S}$. Also, the metallicity residuals for the total metallicity interval, 
Table 6. Statistics for two metallicity calibrations based on new guillotine factors and the ones adopted from Sandage (1969), for two samples: (a) for 75 stars taken from Karaali et al. (2003) and (b) for 469 stars taken from Karataș \& Schuster (2006)

\begin{tabular}{|c|c|c|c|c|}
\hline & \multicolumn{2}{|c|}{ Mean deviation $\langle\mathrm{d}[\mathrm{Fe} / \mathrm{H}]\rangle$} & \multicolumn{2}{|c|}{ Standard deviation $(\sigma)$} \\
\hline & This paper & Sandage (1969) & This paper & Sandage (1969) \\
\hline \multicolumn{5}{|l|}{ (a) $[\mathrm{Fe} / \mathrm{H}](\mathrm{dex})$} \\
\hline$-1.76<[\mathrm{Fe} / \mathrm{H}] \leq-1.0$ & -0.033 & -0.123 & 0.116 & 0.139 \\
\hline$-1.0<[\mathrm{Fe} / \mathrm{H}] \leq-0.5$ & 0.045 & -0.039 & 0.149 & 0.110 \\
\hline$-0.5<[\mathrm{Fe} / \mathrm{H}] \leq 0.0$ & 0.004 & 0.016 & 0.114 & 0.080 \\
\hline $0.0<[\mathrm{Fe} / \mathrm{H}] \leq 0.4$ & 0.009 & 0.054 & 0.081 & 0.084 \\
\hline \multicolumn{5}{|l|}{ (b) $[\mathrm{Fe} / \mathrm{H}](\mathrm{dex})$} \\
\hline$-1.76<[\mathrm{Fe} / \mathrm{H}] \leq-1.0$ & -0.040 & -0.029 & 0.251 & 0.423 \\
\hline$-1.0<[\mathrm{Fe} / \mathrm{H}] \leq-0.5$ & -0.062 & -0.131 & 0.278 & 0.255 \\
\hline$-0.5<[\mathrm{Fe} / \mathrm{H}] \leq 0.0$ & -0.034 & 0.010 & 0.198 & 0.187 \\
\hline $0.0<[\mathrm{Fe} / \mathrm{H}] \leq 0.4$ & 0.036 & 0.095 & 0.166 & 0.177 \\
\hline
\end{tabular}

$-1.76<[\mathrm{Fe} / \mathrm{H}] \leq+0.4$, confirm the advantage of the metallicity-dependent guillotine factors.

For the second set, there is an agreement between the mean deviations for the two calibrations only for the metallicity interval $-1.76<[\mathrm{Fe} / \mathrm{H}] \leq-1 \mathrm{dex}$. The mean deviation of the residuals estimated via $f_{S}$ for the metallicity interval $-0.5<[\mathrm{Fe} / \mathrm{H}] \leq 0$ dex is a bit smaller than the mean deviation of those estimated via $f_{K}$, whereas for the two metallicity intervals $-1<[\mathrm{Fe} / \mathrm{H}] \leq-0.5$ and $0<[\mathrm{Fe} / \mathrm{H}] \leq+0.4$ dex the mean deviations corresponding to $f_{K}$ are much smaller than those of $f_{S}$. In Figure 10 , the residuals estimated via $f_{K}$ and $f_{S}$ are calibrated to linear equations of the metallicities. However, the inclination of the line for Figure 10(a) (0.10) is less than the one for Figure 10(b) (0.20), indicating that the metallicities estimated by means of the calibration based on metallicity-dependent guillotine factors agree better with the original metallicities relative to the other set of estimated metallicities.

We showed that the metallicity-dependent guillotine factors provide more accurate metallicities than those estimated by using the guillotine factors in the literature. This work will be useful for astronomers who work with $U B V$ photometry, which has the advantage of being able to be transformed to other systems.

\section{Acknowledgments}

All the authors are greatful to the anonymous referee whose comments and suggestions improved the paper. $\mathrm{S}$. Karaali is grateful to the Beykent University for financial support. This research has made use of the SIMBAD database, operated at CDS, Strasbourg, France, and the NASA/IPAC Extragalactic Database (NED) which is operated by the Jet Propulsion Laboratory, California Institute of Technology, under contract with the National Aeronautics and Space Administration.

\section{References}

Ak, S., Bilir, S., Karaali, S. \& Buser, R., 2007a, AN, 328, 169 Ak, S., Bilir, S., Karaali, S., Buser, R. \& Cabrera-Lavers, A., 2007b, NewA, 12, 605

Bahcall, J. N. \& Soneira, R. M., 1980, ApJS, 44, 73
Buser, R. \& Kurucz, R. L., 1978, A\&A, 70, 555

Buser, R. \& Kurucz, R. L., 1985, Calibration of fundamental stellar quantities; Proceedings of the Symposium, Como, Italy, May 24-29, 1984 (Dordrecht: D. Reidel Publishing Co.), 513

Buser, R. \& Fenkart, R. P., 1990, A\&A, 239, 243

Buser, R. \& Kurucz, R. L., 1992, A\&A, 264, 557

Cameron, L. M., 1985, A\&A, 152, 250

Carney, B. W., 1979, ApJ, 233, 211

Cayrel de Strobel, G., Soubiran, C. \& Ralite, N., 2001, A\&A, 373,159

Fuhrmann, K., 2008, MNRAS, 384, 173

Hauck, B., Nitschelm, C., Mermilliod, M. \& Mermilliod, J.-C., 1990, A\&AS, 85, 989

Karaali, S., Bilir, S., Karataş, Y. \& Ak, S., 2003, PASA, 20, 165

Karataş, Y.\& Schuster, W., 2006, MNRAS, 371, 1793

Lejeune, Th., Cuisinier, F. \& Buser, R., 1997, A\&AS, 125, 229

Luck, R. E. \& Heiter, U., 2006, AJ, 131, 3069

Marshall, D. J., Robin, A. C., Reylé, C., Schultheis, M. \& Picaud, S., 2006, A\&A, 453, 635

Mishenina, T. V., Soubiran, C., Kovtyukh, V. V. \& Korotin, S. A., 2004, A\&A, 418, 551

Nissen, P. E., Primas, F., Asplund, M. \& Lambert, D. L., 2002, A\&A, 390, 235

Ramirez, I. \& Melendez, J., 2005, ApJ, 626, 446

Roman, N. G., 1955, ApJS, 2, 195

Ryan, S. G. \& Smith, I. M., 2003, MNRAS, 341, 199

Sandage, A. \& Eggen, O. J., 1959, MNRAS, 119, 278

Sandage, A., 1969, ApJ, 158, 1115

Santos, N. C., Israelian, G. \& Mayor, M., 2004, A\&A, 415, 1153

Schlegel, D. J., Finkbeiner, D. P. \& Davis, M., 1998, ApJ, 500, 525

Schwarzschild, M., Searle, L. \& Howard, R., 1955, ApJ, 122, 353

Soubiran, C., Le Campion, J.-F., Cayrel de Strobel, G. \& Caillo, A., 2010, A\&A, 515, A111

Sousa, S. G., Santos, N. C., Mayor, M., Udry, S., Casagrande, L., Israelian, G., Pepe, F., Queloz, D. \& Monteiro, M. J. P. F. G., 2008, A\&A, 487, 373

Spite, M., Francois, P., Nissen, P. E. \& Spite, F., 1996, A\&A, 307, 172

Strömgren, B., 1966, ARA\&A, 4, 433

Tomkin, J. \& Lambert, D. L., 1999, ApJ, 523, 234

Trefzger, Ch. F., Pel, J. W. \& Gabi, S., 1995, A\&A, 304, 381

Valenti, J. A. \& Fischer, D. A., 2005, ApJS, 159, 141

van Leeuwen, F., 2007, ASSL, 350

Vandenberg, D. A. \& Bell, R. A., 1985, ApJS, 58, 561

Wallerstein, G. \& Carlson, M., 1960, ApJ, 132, 276

Wallerstein, G., 1962, ApJS, 6, 407

Walraven, Th. \& Walraven, J. H., 1960, Bull. Astron. Inst. Netherlands, 15, 67

Wildey, R. L., Burbidge, E. M., Sandage, A. R. \& Burbidge, G. R., 1962, ApJ, 135, 94

Yaz, E. \& Karaali, S., 2010, NewA, 15, 234 